\title{
Prolonged Training at Threshold Promotes Robust Retinotopic Specificity in Perceptual Learning
}

\author{
Shao-Chin Hung and Aaron R. Seitz \\ Department of Psychology, University of California-Riverside, Riverside, California 92521
}

\begin{abstract}
Human perceptual learning is classically thought to be highly specific to trained stimuli's retinal location. Together with evidence that specific learning effects can result in corresponding changes in early visual cortex, researchers have theorized that specificity implies regionalization of learning in the brain. However, other research suggests that specificity can arise from learning readout in decision areas or through top-down processes. Notably, recent research using a novel double-training paradigm reveals dramatic generalization of perceptual learning to untrained locations when multiple stimuli are trained. These data provoked significant controversy in the field and challenged extant models of perceptual learning. To resolve this controversy, we investigated mechanisms that account for retinotopic specificity in perceptual learning. We replicated findings of transfer after double training; however, we show that prolonged training at threshold, which leads to a greater number of difficult trials during training, preserves location specificity when double training occurred at the same location or sequentially at different locations. Likewise, we find that prolonged training at threshold determines the degree of transfer in single training of a peripheral orientation discrimination task. Together, these data show that retinotopic specificity depends highly upon particularities of the training procedure. We suggest that perceptual learning can arise from decision rules, attention learning, or representational changes, and small differences in the training approach can emphasize some of these over the others.
\end{abstract}

Key words: perceptual learning; psychophysics; retinotopic specific; transfer of learning; vision

\section{Introduction}

Perceptual learning (PL), experience-induced gains in discriminating sensory features, has classically been thought to be highly specific to the trained retinal location (Karni and Sagi, 1991). Together with evidence that specific learning effects can result in corresponding changes in primary visual cortices (Schoups et al., 2001), researchers have theorized that specificity implies regionalization of learning in the brain (Fahle, 2004). However, other research suggests that specificity in PL can arise from learning readout in decision areas (Dosher and Lu, 1998) or through topdown processes (Li et al., 2008). The issue is more than academic. From a neuroscientific perspective, specificity has been a blessing, providing important clues regarding the neural mechanisms underlying the learning effects. However, from a therapeutic point of view, specificity is a curse because good therapy should generalize broadly to the needs of the patient. Given this, it is not surprising that specificity has been a central focus of the field.

\footnotetext{
Received Feb. 22, 2014; revised May 3, 2014; accepted May 7, 2014.

Author contributions: S.-C.H. and A.R.S. designed research; S.-C.H. performed research; S.-C.H. and A.R.S. analyzed data; S.-C.H. and A.R.S. wrote the paper.

A.R.S. and S.-C.H. were supported by National Science Foundation (BCS-1057625) and National Institutes of Health (1R01EY023582). We thank Cong Yu, Dennis Levi, Stan Klein, and Jun-Yun Zhang for sharing materials and useful conversations regarding this manuscript. We also thank Jennifer Rose, Tina Yang, Kristen Suk, Julian Valdez, Joanne Vuong, and Nick Reihanabad for assistance in conducting the research.

The authors declare no competing financial interests.

Correspondence should be addressed to Aaron Seitz, 900 University Avenue, Riverside, CA 92521. E-mail: aseitz@ucr.edu.

DOI:10.1523/JNEUROSCI.0745-14.2014

Copyright $\odot 2014$ the authors $\quad 0270-6474 / 14 / 348423-09 \$ 15.00 / 0$
}

Recent research, showing that early findings of specificity may not be as reliable as initially observed (Xiao et al., 2008; J.Y. Zhang, 2010, 2011), has generated a great deal of controversy in the field. For example, double training (Xiao et al., 2008) shows that retinotopically specific PL of Vernier acuity or contrast sensitivity can be transferred due to subsequent training with a different stimulus at a different spatial location. These results and others recent studies (Harris et al., 2012) have brought into question numerous findings of low-level neural correlates and fundamentally challenged extant models of PL (Vaina et al., 1998; Schoups et al., 2001; Li et al., 2004; Hua et al., 2010; Adab and Vogels, 2011). While some research provides evidence that transfer after double training may not be ubiquitous (Pilly et al., 2010; Le Dantec and Seitz, 2012), no study has identified when and why double training will lead to transfer and when it will not.

To address this central debate of the field, we investigated mechanisms that account for retinotopic specificity in PL. We replicated double-training enabled transfer of a peripheral Vernier task to untrained locations; however, PL in a different hyperacuity task (i.e., 3-dot hyperacuity task) showed preserved location specificity after double training. We go on to show that prolonged training near threshold, which leads to a greater number of difficult trials during training, rescued location specificity in the Vernier task after double training. To test whether the amount of training at threshold determines location specificity in other paradigms, we show that prolonged training near threshold determines transfer in an orientation discrimination task even without double training. Our results suggest that specificity of learning depends upon particularities of training procedure, which are often ignored. We believe that our study both helps 
settle the debate regarding whether PL involves retinotopically specific mechanisms and it also serves as a call to the field to consider how subtle differences in training can yield dramatic differences in what is learned.

\section{Materials and Methods}

Participants and apparatus. Human subjects of either sex who were naive to research purpose participated and received payment for their participation in experiments. All subjects reported normal or corrected-tonormal binocular visual acuity. Informed consent was obtained from all subjects and experiments were conducted in accordance with the Institutional Review Board approved by the Human Research Review Board of University of California, Riverside.

The stimuli were presented using Psychophysics Toolbox (Brainard, 1997; Pelli, 1997) for MATLAB (MathWorks) on a Mac mini computer with a 24 inch SonyTrinitron CRT monitor with resolution of $1600 \times$ 1024 pixels and a refresh rate of $100 \mathrm{~Hz}$. A ViewPoint Eye Tracker system running at $220 \mathrm{~Hz}$ (USB-220; Arrington Research) and head positioner including chin rest were used to ensure eye fixation at the center of the display throughout each trial in the experimental sessions. The configuration of the eye tracking system was displayed on a PC and controlled via an Ethernet communication between PC and Mac. All experiments (except the last experiment that only used the orientation discrimination task) were performed with a gaze-contingent display in which the eye tracker enabled new trials to start only when subjects fixated at the center of the screen (within a 2 degree radius fixation window). If an eye movement outside of this window was detected at any point after the trial started, then that trial was aborted (and ignored in the analysis) and a new trial was initiated.

Stimuli. Stimuli used were a pair of identical Gabor patches (Gaussian windowed sinusoidal gratings) in the Vernier task and a single Gabor patch in the orientation task presented at $5^{\circ}$ retinal eccentricity on a gray background. The Gabor in the orientation discrimination task had spatial frequency of $3 \mathrm{cpd}, \mathrm{SD}$ of $2 \lambda$, and contrast of 0.45 . In addition, the two Gabors in the Vernier task had a center-to-center distance of $4 \lambda$, with other parameters being the same as described in the orientation task. The position of Vernier stimuli jittered $0.25^{\circ}$ eccentricity (horizontally for vertical stimuli and vertically for horizontal stimuli) across trials to prevent subjects from using external cues such as the edge of monitor to judge stimuli's offset.

For the three-dot hyperacuity task, a white vertical 3-dot stimulus was presented at $7.5^{\circ}$ retinal eccentricity on a black background. Each dot had a radius of $2 \mathrm{~min}(\mathrm{arcmin})$, and the distance between the first and third dot was $20 \mathrm{~min}$. Each trial consisted of one aligned 3-dot stimulus, and one offset stimulus with the middle dot offset to the right or left. We used offset variables that represented five different difficulties $(0.9,1.8,2.7$, 3.6 , and $4.5 \mathrm{~min}$ ). The viewing distance was 59 inches in all experiments that used an eye tracker and 30 inches in the orientation experiment without an eye tracker.

Procedure. In the Vernier task, Vernier stimulus was presented for 200 ms, and the subject's task was to judge whether the lower Gabor was to the right or left versus the upper one for a vertical Gabor pair, or whether the right Gabor was higher or lower than the left for a horizontal Gabor pair. In the orientation discrimination task, stimuli were presented in two intervals ( $92 \mathrm{~ms}$ each) separated by an interstimulus interval of 600 ms. One reference stimulus $\left(36^{\circ}\right.$ or $\left.126^{\circ}\right)$ and one target stimulus (reference + clockwise offset) were presented in a sequential order, and subjects were asked to indicate whether the first or the second interval contained the target stimulus. A central fixation cross was presented for $400 \mathrm{~ms}$ before stimulus onset in both tasks and stayed through the trial. Both tasks were performed under a 3-down-1-up staircase rule and the step size was 1 arcmin in the Vernier task and 1 degree in the orientation task. All stimulus parameters and the step size in the orientation task were the same as in previous publications (Xiao et al., 2008; J. Y. Zhang et al., 2010) to provide comparable comparison between two labs' results. Each block contained 20 reversals or 100 trials depending on whichever reached first, and the threshold was calculated from the last 10 reversals in each block.
For the $7 \mathrm{~d}$ study, subjects were tested on vertical or horizontal Vernier stimuli at each of four quadrants (one block each condition) on their first and last day and received five double-training sessions on days 2-6. Double training of Vernier and orientation tasks was initiated by the Vernier task and followed by the orientation task in alternating blocks (eight blocks for each task). Threshold in the training session was only acquired from the first two blocks (first block of each task) to avoid contamination from within-session learning (Sotiropoulos et al., 2011) and to provide comparable data to that in testing sessions. This also ensured that the threshold estimate was comparable between the short and long staircase conditions.

In the 3-dot hyperacuity task, each trial began with a fixation period of $300 \mathrm{~ms}$ followed by the stimuli presentation. Two stimuli, one aligned and one offset 3-dot (50 ms each), were presented successively separated by a $400 \mathrm{~ms}$ interstimulus interval. Subjects had to indicate whether the first stimulus or the second one was offset. Threshold in this task was estimated by a power function $\left(f(x)=a x^{n}\right.$, where $a$ is a constant and $n$ is a real number) where subjects achieved $75 \%$ accuracy.

The testing session of 3-dot hyperacuity task consisted of 800 trials equally distributed between eight different conditions (e.g., 3-dot stimulus with left or right offset at each of four quadrants). The 100 trials in each condition were divided into 20, 5 trial mini-blocks; 4 mini-blocks of each of the 5 offset sizes. Blocks of 50 trials ( 10 mini-blocks, two for each offset size) were randomized across locations and breaks were given between blocks.

The double-training session of the 3-dot hyperacuity task consisted of 400 trials in the same condition (i.e., single location and orientation) divided into eight blocks (50 trials each block with 10 trials per offset size), with each block followed by one block of the orientation task.

In the experiment that only used the orientation identification task, subjects received a short $(5 \mathrm{~min})$ practice session $1 \mathrm{~d}$ before the testing session. In the testing session they were tested on a single Gabor patch at the upper left and lower right locations (two blocks each). The testing session had 20 reversals in each block while the training session, which consisted of 16 blocks, had 10 reversals. Threshold in the testing session was analyzed from the last 10 reversals and was determined by the average of the fifth to the tenth reversals from the first two blocks in the training session. This method was consistent with Xiao et al. (2008); however, the qualitative pattern of results' statistical significance was resilient to other methods of threshold calculation.

In all experiments, subjects had $2 \mathrm{~s}$ to answer by a key press, and feedback was given as a green cross flashed at the center of the screen for correct responses, or a red central cross for incorrect responses. If subjects failed to respond ( $1.8 \%$ of trials on pretest and $1.1 \%$ of trials on post-test), the message "You didn't respond in time. Please wait for the next trial" was presented for $2 \mathrm{~s}$ before the next trial commenced. The location and orientation of trained stimuli were counterbalanced across subjects.

\section{Results}

We first replicated the most counterintuitive example of double training, namely the "piggybacking effect," where learning of a peripheral Vernier hyperacuity task transfers to another spatial location after training an orientation discrimination task at the same location (Zhang et al., 2011). The Vernier hyperacuity task has traditionally been considered as a location-specific task (Xiao et al., 2008) and so we first attempted an exact replication of the piggybacking effect. We did so under very tight experimental control, using an eye tracker to create gaze-contingent displays where trials were aborted as soon as subjects made any eye movement. In this way, we assured that training and testing stimuli were always at the intended retinotopic locations (Hung and Seitz, 2011). Six subjects participated this $7 \mathrm{~d}$ study and were tested on Vernier stimuli at the trained and untrained locations (see above, Procedure) on their first and last day. On days 2-6, they performed five double-training sessions ( $2 \mathrm{~h}$ each day), which consisted of Vernier and orientation discrimination tasks 
A

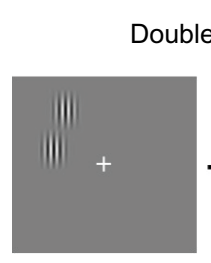

Ver_loc1
Double Training

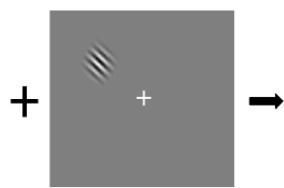

Ori_loc1
B

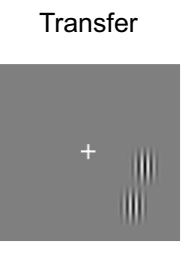

Ver_loc2

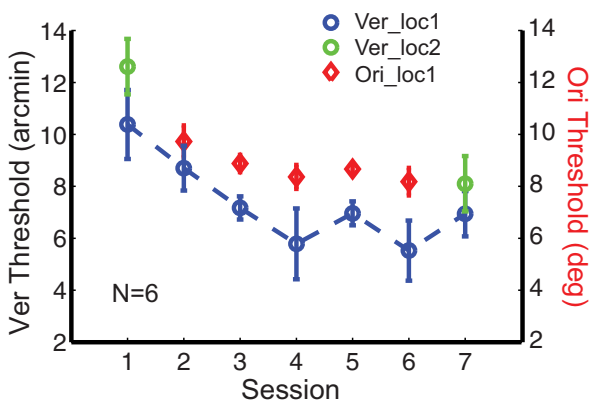

C

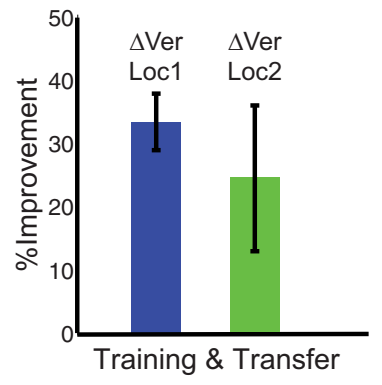

Figure 1. Transfer of Vernier learning after double training at the same location. $\boldsymbol{A}$, Stimulus configuration. Double training of Vernier and orientation discrimination tasks was performed at the same location (loc1), and transfer of Vernier learning was tested at the untrained diagonal location (loc2). $\boldsymbol{B}$, Mean session-by-session threshold analysis for Vernier learning (blue circles) and orientation learning (red diamonds). Transfer of Vernier learning was tested before and after double training (green circles). The practice of location-unspecific orientation learning piggybacked Vernier learning to the untrained location. C, MPI for Vernier learning at the trained (Loc1, blue bar) and untrained (Loc2, green bar) location. Vernier learning transferred to the untrained location (Loc2) after double training. Error bars represent within-subject SE.

A B

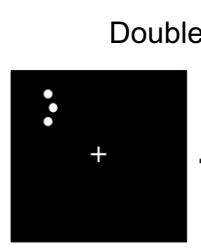

Dot_loc1

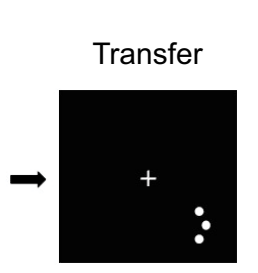

Dot_loc2
C

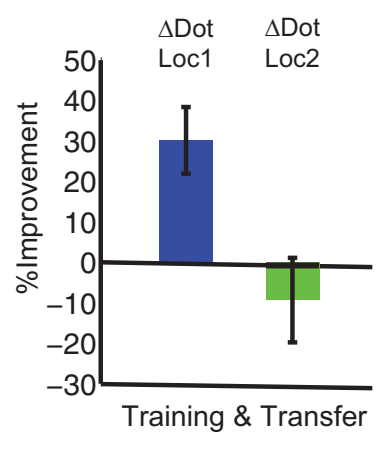

Figure 2. Retinotopic specificity in the 3-Dot hyperacuity task after double training. $\boldsymbol{A}$, Double training of 3-dot hyperacuity and orientation discrimination tasks was performed at the same location (loc1), and transfer of 3-dot hyperacuity learning was tested at the untrained diagonal location (loc2). $\boldsymbol{B}$, This plot shows session-by-session thresholds for 3-dot hyperacuity learning (blue circles) and orientation learning (red diamonds) and transfer results of 3-dot hyperacuity task (green circles). Improvement of the 3-dot hyperacuity task did not transfer to the untrained location (green circles). C, MPI of 3-dot hyperacuity learning at the trained (Loc1, blue bar) and untrained (Loc2, green bar) location is shown. The 3-dot hyperacuity task retained retinotopic specificity after double training.

at the same location in alternating blocks (Fig. 1A). After training, we found significant learning in the Vernier task at the trained location (Fig. $1 B, C ; \Delta$ Ver_loc1, Mean Percent Improvement $[\mathrm{MPI}]=33.5 \pm 4.5 \%, p=0.003$, one-tailed, paired, $t$ test $)$, with a gradual, nonsignificant learning in the orientation task ( $\Delta$ Ori_loc1; MPI $=16.1 \pm 11.5 \%, p=0.12$ ). More importantly, Vernier learning also transferred to the untrained orthogonal location $(\Delta$ Ver_loc2, MPI $=24.5 \pm 11.6 \%, p=0.04)$. These data confirm the piggybacking effect and suggest a broad spatial transfer of feature learning (e.g., Vernier task) when paired with a second, location-unspecific training (e.g., orientation task) at the same location.

To examine how ubiquitously double training can lead to retinotopic transfer, we examined the piggybacking effect using a different hyperacuity task (i.e., 3-dot hyperacuity task). Seven subjects participated in this experiment in which they performed alternating blocks of a 3-dot hyperacuity task and the orientation task, both trained at the same location (Fig. 2A). Notably, while we found significant learning in both the 3-dot hyperacuity task and the orientation task (Fig. $2 B, C$; $\Delta$ Dot_loc1, MPI $=30.2 \pm$ $8.3 \%, p=0.007$ and $\Delta$ Ori_loc1, MPI $=31.3 \pm 7.8 \%, p=0.003$ ), $\mathrm{PL}$ in the 3-dot hyperacuity task did not transfer to the untrained quadrant $(\Delta$ Dot_loc2, MPI $=-9.4 \pm 10.5 \%, p=0.67)$. These results demonstrate that double training does not ubiquitously lead to retinotopic transfer in PL.

We next sought to understand why double training led to transfer in the Vernier task but not in the 3-dot hyperacuity task. We observed that Vernier training used multiple short staircases, while the 3-dot hyperacuity training used the method of constant stimuli. This methodological difference, in which the former included many easy trials (above threshold), while the latter included many difficult trials (at or below threshold), leads to a difference in task difficulty and stimulus precision (i.e., stimuli with small offset difference) during the training of the two tasks. Of note, while these repeated short staircases are typical of the double-training studies that found transfer (Xiao et al., 2008), many studies finding specificity used prolonged training at threshold, typically with a single staircase per session (Schoups et al., 1995; Jehee et al., 2012; Le Dantec and Seitz, 2012). We thus hypothesized that the difficulty/precision of the training stimuli may be key to driving specificity. While this hypothesis has never been investigated in the context of double training, it is consistent with other results in the literature showing that task difficulty during training (Ahissar and Hochstein, 1997) or precision of the transfer task (Jeter et al., 2009) shapes the specificity of PL. 
A

Multiple Staircases

Single Staircase
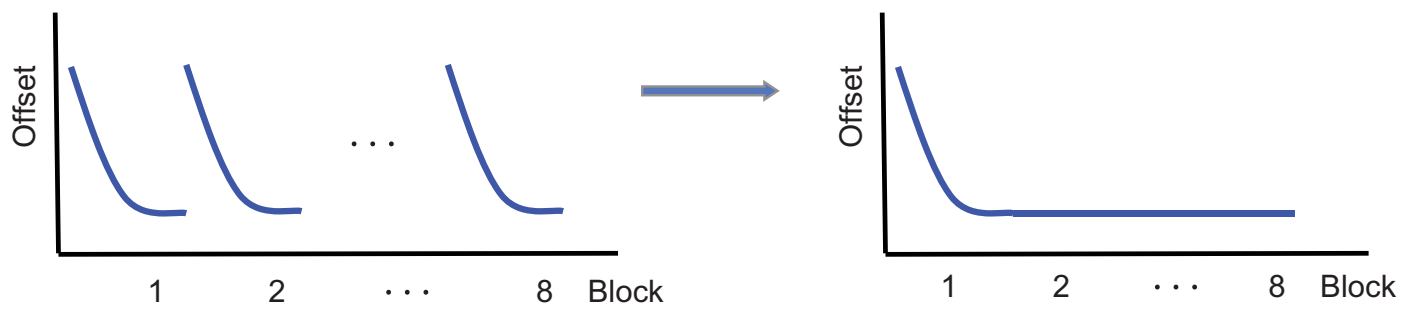

B
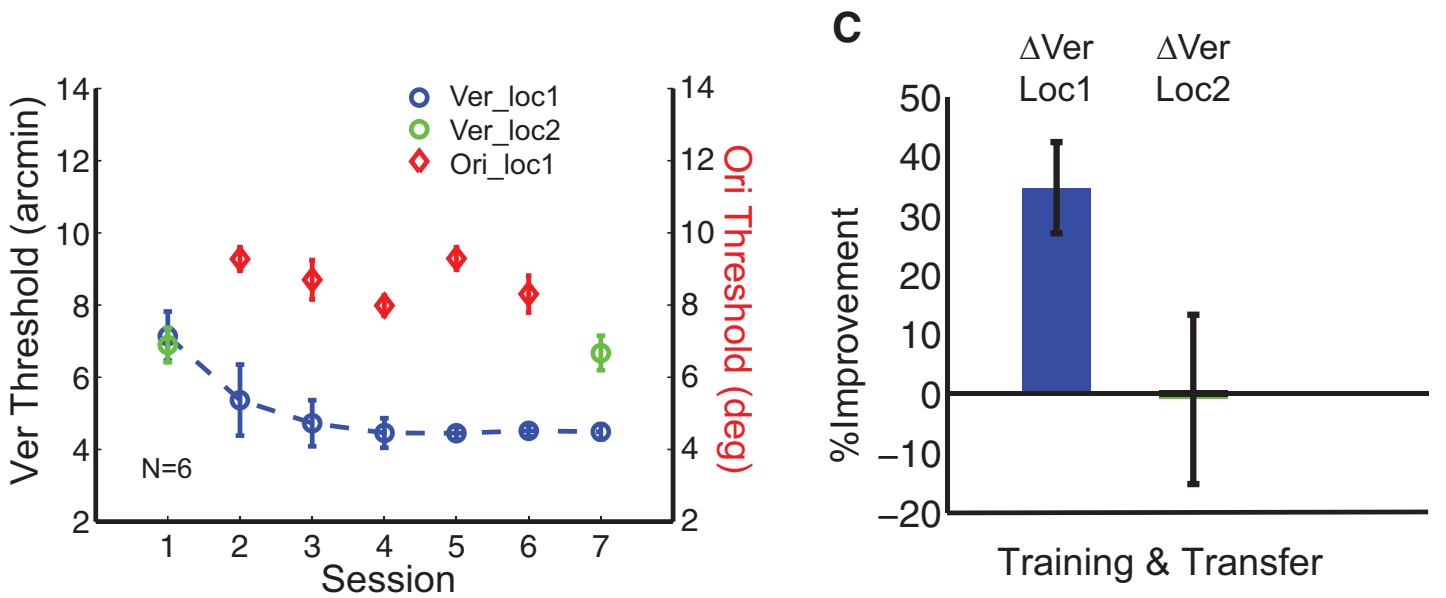

Figure 3. Training with a single staircase per session restored spatial specificity of Vernier learning. $A$, Comparison between the multiple short staircases and the single staircase. Conventional multiple staircases return back to stimuli's original offset and contain many easy trials as the new block begins. The single staircase remains around subjects' threshold for almost the entire training session. $\boldsymbol{B}$, Session-by-session threshold analysis for Vernier learning (blue circles) and orientation learning (red diamonds) in the single staircase condition. Transfer of Vernier learning was shown in green circles. Double training at the same location in the single staircase condition failed to produce transfer of Vernier learning to the untrained location (green circles). $\boldsymbol{C}$, Percentage improvement of Vernier learning at the trained (Loc1, blue bar) and untrained (Loc2, green bar) locations in the single staircase condition.

To test this hypothesis, we replaced the repeated short staircases, which contain a larger portion of trials above threshold, used in the Vernier training with a single staircase in which a larger portion of trials are at threshold (Fig. $3 A$ ). The staircases used in the testing sessions were identical to that of repeated short staircase condition, and the threshold estimates during training were from the same period at the beginning of each session to make the data as comparable as possible. Six subjects were recruited and trained in the single staircase condition with the experimental paradigm exactly the same as in Figure $1 A$. Here we found that prolonged training at threshold restored location specificity in the Vernier task (Fig. $3 B, C$; $\Delta$ Ver_loc1, MPI = $34.7 \pm 7.7 \%, p=0.008$ and $\Delta$ Ver_loc 2 , MPI $=-1.0 \pm 14.2 \%$, $p=0.41)$. These results are in stark contrast to those found in the Vernier task trained with multiple short staircases, which lead to a limited amount of training at threshold, even though all other aspects of the experiment were preserved. These results support our hypothesis that PL with more difficult tasks/precise stimulus discriminations is more likely to retain specificity, even under double training.

To confirm the finding that prolonged training at threshold influences transfer in PL, we replicated these results by applying the single staircase to the two-location sequential double-training paradigm that was first reported by Xiao et al. (2008). Eleven new subjects participated in this $13 \mathrm{~d}$ study and were sequentially trained on two different orientations of the Vernier stimuli, each at a different location (Fig. $4 A$ ). In the first stage of double train- ing (i.e., days 2-6), they were trained with orientation 1 at location 1 (ori1_loc1), and in the second stage (i.e., days 8-12) were trained with the orthogonal orientation at the diagonal location (ori2_loc2). On days 1, 7, and 13, subjects received pre-, mid-, and post-training testing sessions, respectively. All training sessions used the single staircase method. After successive training at ori1_loc1 (Fig. 4B, blue circles; $C$, left blue bar; MPI $=34.1 \pm$ $7.7 \%, p<0.001$ ), contrary to prior results (Xiao et al., 2008), sequential double training did not lead to improvement of performance at ori1_loc2 after training on ori2_loc2 (Fig. 4B, the second and third green circles; $C$, right green bar; MPI $=-4.2 \pm$ $9.2 \%, p=0.62)$. However, when all training sessions used multiple short staircases (Fig. $4 D, E$ ), improvement of performance took place at ori1_loc2 (Fig. $4 D$, the second and third green circles; $E$, right green bar; MPI $=18.7 \pm 10.5 \%, p=0.02$ ).

Of note, to examine transfer unlocked by the second training, for this replication (Xiao et al., 2008) with short-staircases, subjects who showed significant transfer at the mid-test $(n=5)$ were dropped from the study (see next paragraph) to avoid the potential confound that lack of transfer from double training would be due to a ceiling effect generated from transfer at the mid-test. As such, the data shown in Figure $4 D$ is most comparable to that in Figure $5 \mathrm{~A}$ given that these two subject groups had identical selection criteria at the mid-tests. Additionally, comparing the degree of location transfer between the mid-test and post-test in the short and single staircase conditions, the results showed a significant difference (Fig. $4 C, E$, right green bars; $p=0.01$, one-tailed, 
A

Primary Training

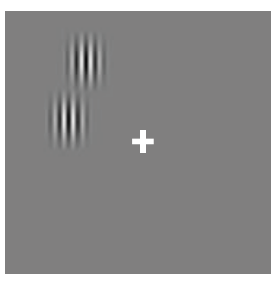

ori1_loc1

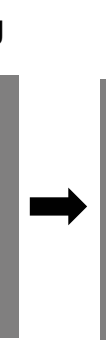

-

ori1_loc2
Mid-test

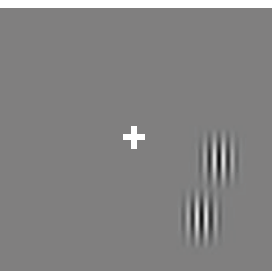

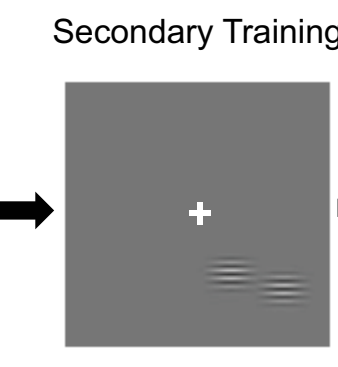

ori2_loc2
Post-test

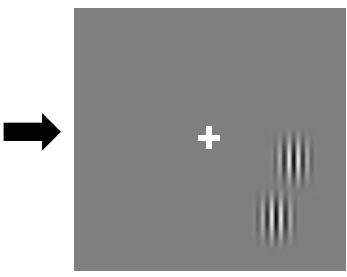

ori1_loc2
B

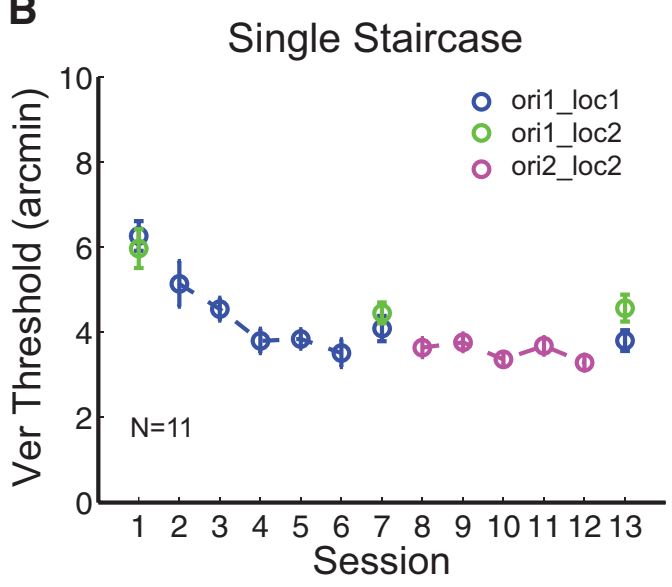

C

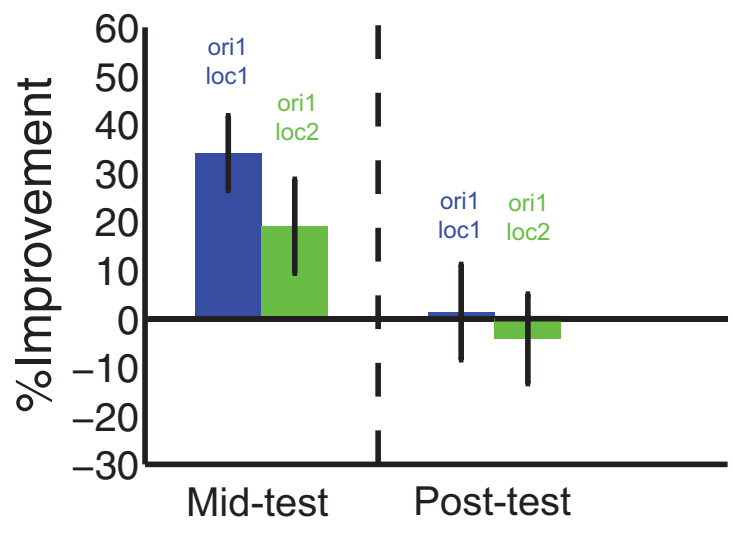

D

\section{Multiple Short Staircases}

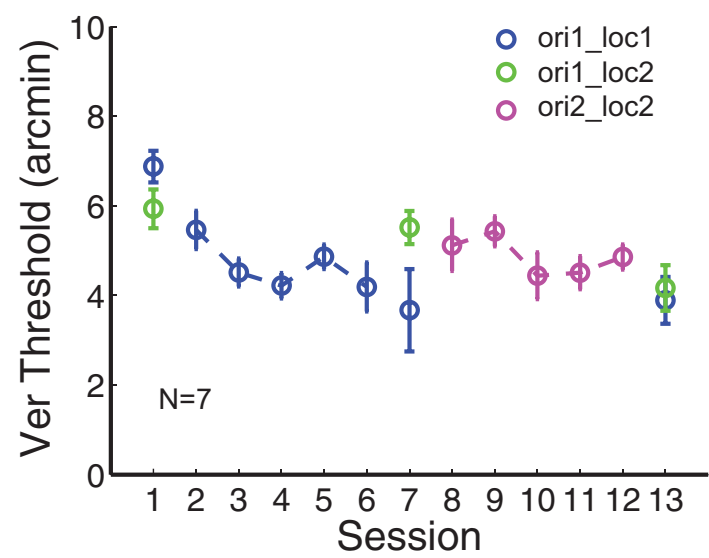

E

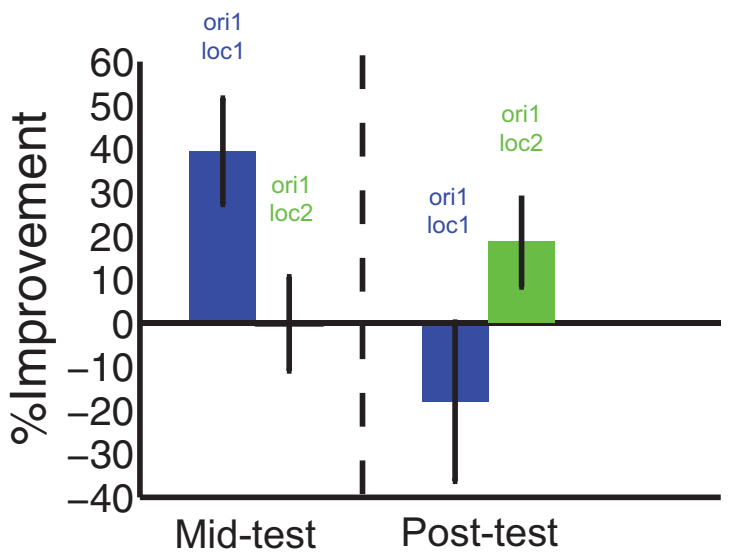

Figure 4. Sequential double training in Vernier discrimination. $A$, Schematic drawing showing sequential double training. $B, C$, Sequential double training in the single staircase condition. Eleven subjects were first trained with Vernier stimuli at ori1_loc1 (blue circles) and then sequentially at ori2_loc2 (magenta circles). Transfer of Vernier learning was measured at ori1_loc2 in pre-, mid-, and post-testing sessions (green circles). Sequential location training (ori2_loc2) did not induce transfer of Vernier learning to untrained locations (ori1_loc2, second and third green circles in $\boldsymbol{B}$; right green bar in C). Bars in the mid-test and post-test indicate threshold differences between the pre-versus mid-test, and mid-versus post-test, respectively. $\boldsymbol{D}, \boldsymbol{E}$, Sequential training in multiple short staircase condition. Contrary to $\boldsymbol{A}$ and $\boldsymbol{B}$, sequential location training (ori2_loc2) enabled transfer of learning to ori1_loc2 in this multiple short staircase method (the second and third green circles in $\boldsymbol{D}$; right green bar in $\boldsymbol{E})$.

unpaired, $t$ test). These data further support our hypothesis that prolonged training at threshold leads to retention of retinotopic specificity when subjected to double training.

A complexity in our data is that we observed substantial transfer of learning to the untrained location in the mid-testing session (Fig. $4 B$, first two green circles; $C$, left green bar; MPI $=19 \pm$ $9.7 \%, p=0.01)$. Further observation of the data indicated that there were significant individual differences in the degree of specificity observed after ori1_loc1 training, an effect reported by Zhang et al. (2013). Thus, to determine whether the lack of transfer under double training was resultant from the level of initial transfer, we split the 11 subjects in Figure $4 B$ into a "specificity" group $(N=6)$ and a "transfer" group $(N=5)$ based upon the retinotopic transfer observed in the mid-testing session (Fig. 5). 
A
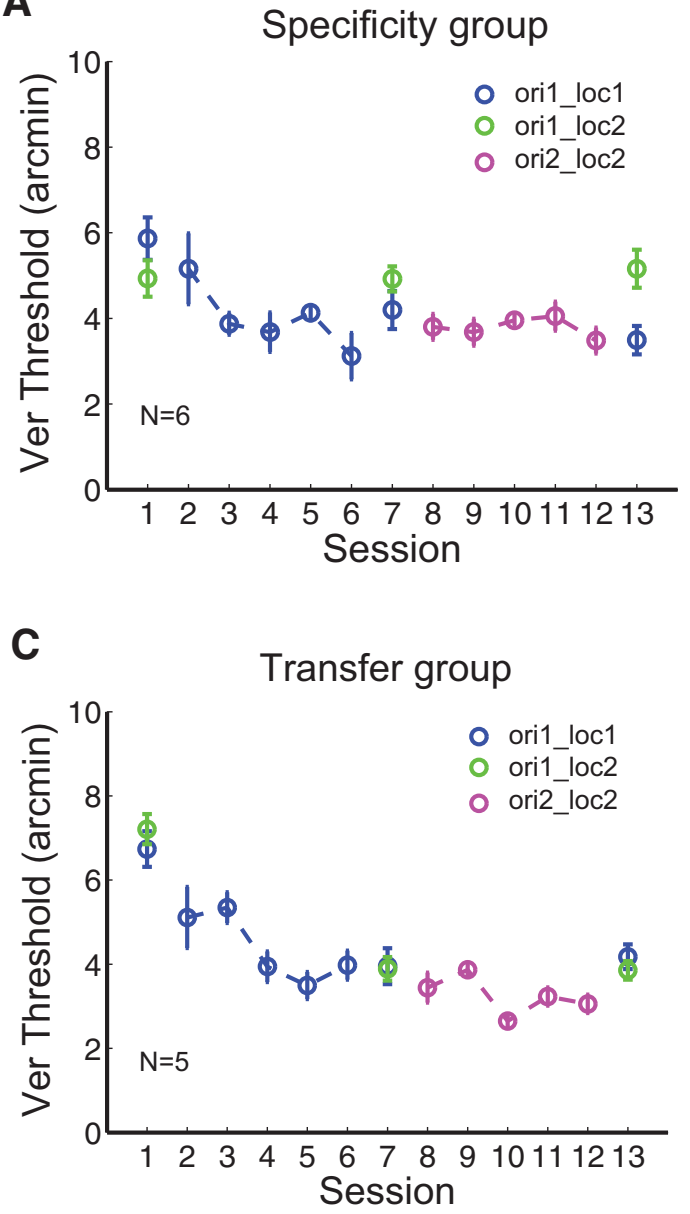

B
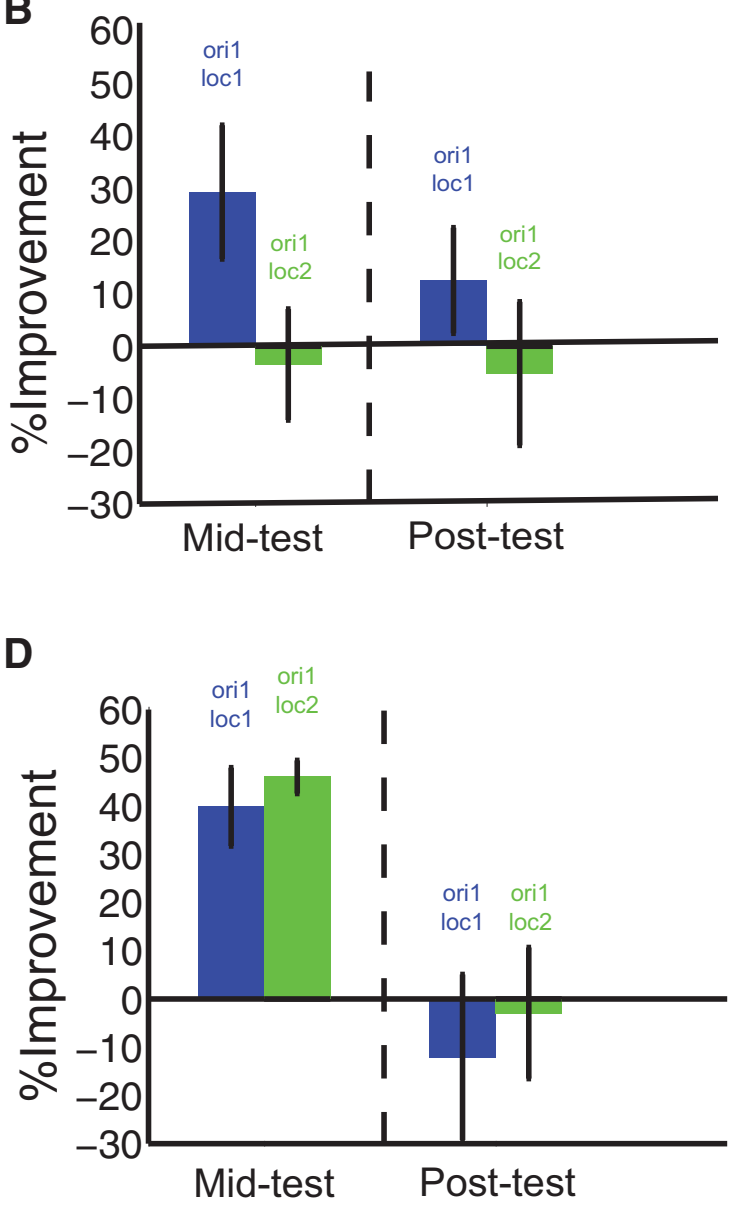

Figure 5. Specificity and transfer groups in the single staircase condition in sequential double training. $\boldsymbol{A}, \boldsymbol{B}$, Sequential Vernier training in specificity group. Vernier learning showed location specificity in this group (first two green circles in $\boldsymbol{A}$; left green bar in $\boldsymbol{B}$ ). Double training did not enable transfer of Vernier learning (ori1_loc1) to ori1_loc2 (second and third green circles in $\boldsymbol{A}$; right green bar in $\boldsymbol{B}$ ). $\boldsymbol{C}, \boldsymbol{D}$, Sequential Vernier training in transfer group. Initial training of ori1_loc1 resulted in transfer of learning to ori1_loc2 (first two green circles in $\boldsymbol{C} ;$ left green bar in $\boldsymbol{D}$ ). However, the second location training (ori2_loc2) did not promote additional transfer to ori1_loc2 (the second and third green circles in $\boldsymbol{C}$; right green bar in $\boldsymbol{D}$ ).

A transfer index (TI) was calculated as the MPI at the untrained location ( $\Delta$ Ver_ori1_loc2) divided by the MPI at the trained location ( $\Delta$ Ver_ori1_loc1). The specificity group (Fig. $5 A, B$ ) had $\mathrm{TI}=-0.12$ indicating no spatial transfer, and the transfer group (Fig. $5 C, D$ ) had TI $=1.16$, which indicates complete transfer. We next investigated whether the degree of transfer induced by double training was consistent between these two groups. We found that neither the specificity group nor the transfer group showed any measureable, additional transfer to the second location after sequential double training (Fig. $5 A, C$, the second and third green circles). MPIs were $-5.2 \pm 13.7 \%(p=0.67)$ and $-2.9 \pm 13.6 \%$ $(p=0.47)$ for the specificity group and the transfer group, respectively (Fig. $5 B, D$, green bars in the post-test).

An important question is whether prolonged training at threshold also determines location specificity in other paradigms in addition to double training. For example, there is significant controversy regarding location specificity of peripheral fine orientation discrimination even under single training (T. Zhang et al., 2010). While classic studies show that PL of orientation discrimination is highly retinotopically specific (Schoups et al., 1995), recent studies show that changing simple parameters, such as an introduction of a pretest, can lead to location transfer (T. Zhang et al., 2010). This is despite the fact that single-unit recording in monkeys (Schoups et al., 2001) and fMRI in humans (Jehee et al., 2012) show plasticity in V1 related to such training (but see Ghose et al., 2002).

Noting different methods used across these studies (Schoups et al., 1995; T. Zhang et al., 2010) we hypothesized that stimulus precision during training may explain divergent results regarding location specificity in a peripheral orientation task. To test this, 17 subjects participated in this $7 \mathrm{~d}$ experiment and were randomly assigned to two different training conditions: multiple short staircases $(N=8)$ or a single staircase $(N=9)$. On days 1 and 7 , subjects were tested on two diagonal locations (upper left and lower right) and in between two testing sessions, they received five training sessions ( $1 \mathrm{~h}$ each day) performed at a single location (Fig. 6A). We found that subjects in both conditions demonstrated significant learning on the trained location (Fig. $6 C$, MPIs were $31.6 \pm 5.9 \%, p=0.002 ; E, 53.2 \pm 3.8 \%, p<$ 0.001 ), with varying amounts of transfer on the untrained location (Fig. 6C,E, green bars). This may be due to the inclusion of a pretest, which has been shown to promote transfer in other studies of peripheral orientation discrimination (T. Zhang et al., 2010). However, there was significantly greater transfer in the multiple staircases condition compared with the single staircase condition (Fig. $6 C, E, p=0.03$, one-tailed, unpaired, $t$ test). These results thus suggest that prolonged training at threshold impacts the specificity of PL even in the absence of double training. 
A

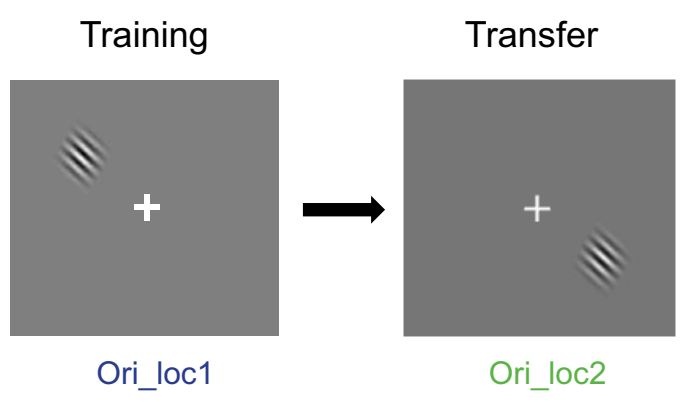

B

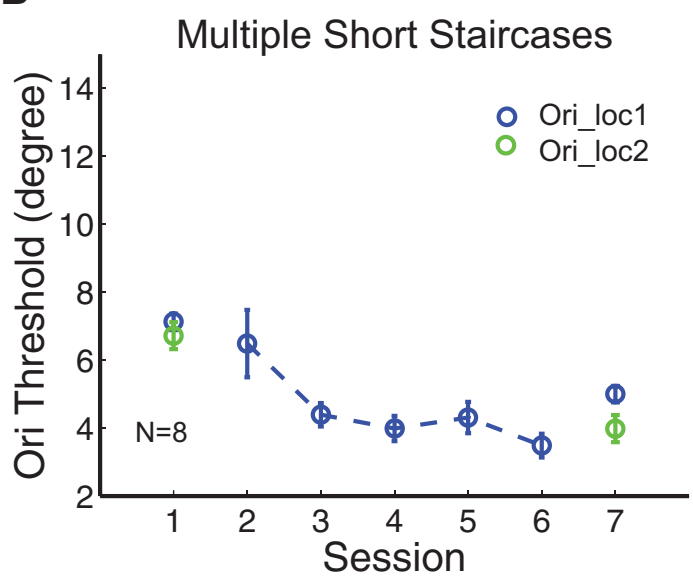

C

D

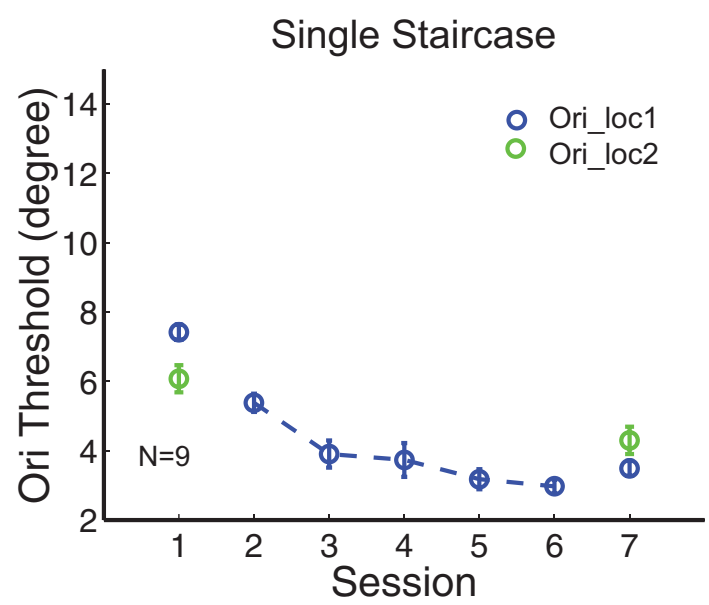

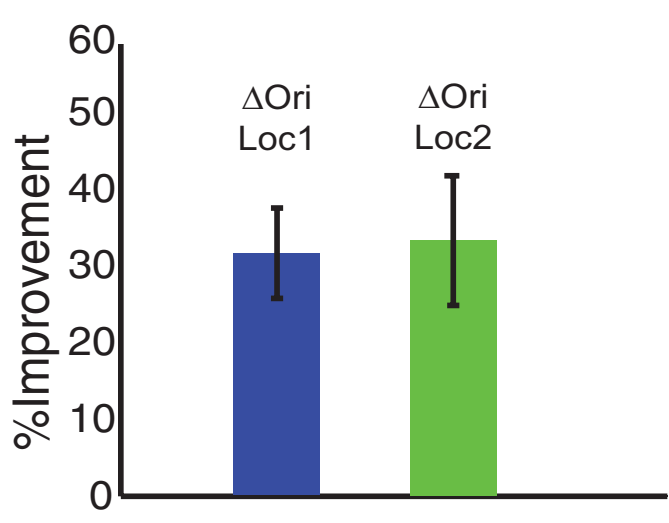

E

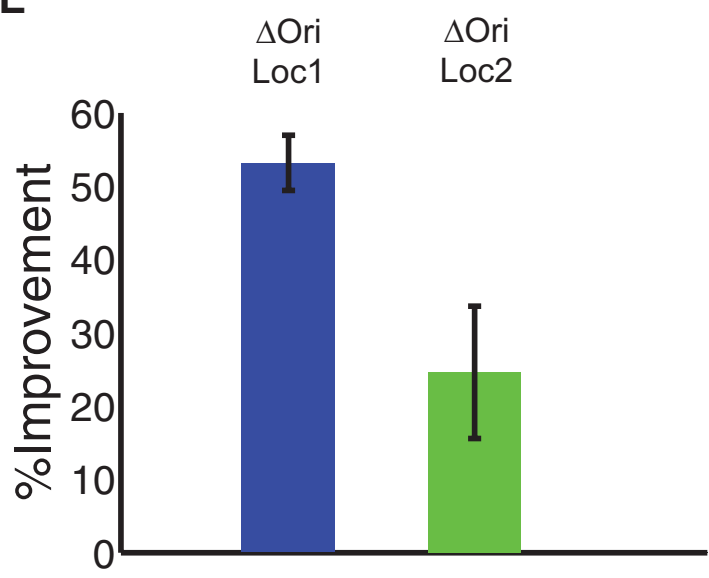

Figure 6. Results of the orientation discrimination task in multiple short staircase condition $(\boldsymbol{B}, \boldsymbol{C})$ and single staircase condition $(\boldsymbol{D}, \boldsymbol{E})$. $\boldsymbol{A}$, Stimulus configuration. The orientation discrimination task was performed at loc1, and transfer of learning was tested at the untrained diagonal location (loc2). $\boldsymbol{B}$, C, Session-by-session threshold analysis and a summary of MPI for the orientation learning used with multiple short staircases. Transfer of learning was shown in green circles in $\boldsymbol{B}$ and the green bar in $\boldsymbol{C}$. The amount of transfer at the untrained diagonal location (green bar) was equivalent to learning at the trained location (blue bar). $\boldsymbol{D}, \boldsymbol{E}$, Results in the single staircase condition. The same training procedure did not promote transfer comparable to $\boldsymbol{C}$ when the task difficulty was highly increased.

Together, we found no evidence that either simultaneous or sequential double training induced retinotopic transfer of $\mathrm{PL}$ in the single staircase condition. As such, retinotopic specificity of PL is not ubiquitously undone by double training and instead depends on the proportion of difficult trials during training.

\section{Discussion}

In this study, we provide a simple and elegant solution to when and why training led to location transfer in these tasks and when it did not. We first replicated the most counterintuitive example of double training, namely the piggybacking effect, in which peripheral hyperacuity of a Vernier task transfers to another spatial location after training an orientation discrimination task at the same location (Zhang et al., 2011). We then showed that this piggybacking effect is not ubiquitous, even to hyperacuity stimuli, by showing that the same secondary task (i.e., an orientation task) did not induce any location transfer for the 3-dot hyperacuity task. To elucidate the mechanism of learning specificity, we examined how prolonged training at threshold during training, which increases the proportion of difficult/precise stimuli, led to 
preserved retinotopic specificity in the Vernier task after double training. These experiments were done under a gaze-contingent display, since subjects' eye movements can confound experimental results when a task is performed in the periphery (Hung and Seitz, 2011). We then validated these findings to other paradigms by showing that prolonged training at threshold also determines the amount of transfer under single training of peripheral fine orientation discrimination. While previous studies have shown that task difficulty (Ahissar and Hochstein, 1997, 2004) and stimulus precision (Jeter et al., 2009) help determine the degree of specificity of PL, to date there has been no evidence that proportion of difficult/precise stimuli during training interacts with other methodological manipulations such as double training or pretraining to determine retinotopic specificity.

A consideration is whether there is a relationship between the degree of learning in the transfer-inducing task and the amount of transfer that is unlocked by double training. Of note, in Figures $1 B$ and $3 B$, learning of the orientation identification task was improved but not significantly so. To test if there was a relationship between learning in the orientation task and the extent of transfer, we compared the correlation between the degrees of orientation learning and transfer of the Vernier task. There was no significant correlation between these variables $(r=0.09, p=$ 0.73 ). Further analysis also revealed no correlation between improvements of the transfer-inducing task (i.e., orientation task in Figs. 1-3 and Vernier task in orthogonal orientation and location in Fig. 4) and transfer of the feature task $(r=0.14, p=0.47)$. Another concern is whether the blockwise training of two different tasks would interfere with results due to the roving. However, given that roving is ineffective if each stimulus is presented for five or more consecutive trials (Zhang et al., 2008) and each block contained 80-100 trials, this should not be a factor that confounds our study.

It is notable that there were substantial individual subject differences in the degree of transfer after initial training, even when training with the single staircase (Fig. 5). Individual differences in the degree of transfer have often been observed in studies of PL (Aberg and Herzog, 2009), and the degree of transfer can depend upon the amount of training, with shorter training periods producing more transfer (Jeter et al., 2010). Notably, the portion of subjects in our transfer group versus the specificity group are comparable to the psychophysical data from a recent ERP study examining mechanisms underlying these individual subject differences using the same training parameters (Zhang et al., 2013). However, critical to the point of the current study, the degree of initial transfer was independent of the effects of double training.

Our results provide a powerful demonstration that subtle changes to the training procedure can result in profound differences in the observed learning effects. To answer the central debate in the field about whether PL is due to representational changes (Karni and Sagi, 1991; Schoups et al., 2001; Yotsumoto et al., 2008; Adab and Vogels, 2011), decision rules (Xiao et al., 2008; J. Y. Zhang et al., 2010; Kahnt et al., 2011), or weight changes in readout (Dosher and Lu, 1998; Law and Gold, 2008), etc., we suggest a simple answer: all of the above. However, importantly, the distribution of learning across the neural system depends upon the fine details of the training procedure. Changing the difficulty/precision of the stimuli (Ahissar and Hochstein, 1997; Jeter et al., 2009), whether a pretest is used (T. Zhang et al., 2010), whether multiple stimuli are trained (Xiao et al., 2008), or the degree to which stimuli do or do not promote adaptation (Harris et al., 2012), have each been shown to dramatically influ- ence the degree to which PL is specific to the trained retinotopic location. Likely, numerous other undiscovered methodological details [such as types of stimuli used (Pilly and Seitz, 2009), design of training/testing sessions, environmental settings, subject instructions, etc.] also play roles in determining the characteristics of PL (unfortunately even those that some researchers think not important to report in their papers).

Our demonstration that prolonged training at threshold promotes specificity of PL builds upon a foundation of other results, indicating that the distribution of plasticity across the visual system is mediated by the sensitivity of the neurons in the brain regions to distinguish the relevant stimuli. For example, the Reverse Hierarchy Theory (Ahissar and Hochstein, 1997, 2004) suggests that harder trials trigger early input levels and make learning more specific to trained features in visual PL. Training with finer stimulus discriminations increased task difficulty and thus is more likely to have neurons tuned to the trained location, resulting in specificity. Similarly, the Integrated Reweighting Theory proposed by Dosher et al. (2013), which explains location transfer by incorporating higher level, location-independent representations into a multilevel learning system, includes the feature that the lower level, location-specific representations have narrower orientation tuning functions and less noisy responses. In this model, double-training procedure may weight more locationindependent representations to the decision unit and thus results in location transfer, while increased stimulus precision reweights location-specific representations and thus restores location specificity of the task. As such, we believe that the ratio of precise to imprecise stimuli during training, and not the total number of precise stimuli, will determine transfer; however, further research will be required to evaluate empirically this prediction. Together these models suggest a consistent view that the brain structures that are most diagnostic to solve the task are those that learning should most rely upon to get better and these models help explain why prolonged training at threshold may help determine the locus of plasticity in the brain.

We believe that our study both helps settle the debate regarding whether PL involves retinotopically specific mechanisms (i.e., it can or cannot, depending on the training methods) and serves as a call to the field to consider how subtle differences in training can yield dramatic differences in what is learned. To advance our understanding of PL, the field must move toward understanding individual, and procedurally induced, differences in learning and how multiple neural mechanisms may together underlie behavioral learning effects.

\section{References}

Aberg KC, Herzog MH (2009) Interleaving bisection stimuli-randomly or in sequence-does not disrupt perceptual learning, it just makes it more difficult. Vision Res 49:2591-2598. CrossRef Medline

Adab HZ, Vogels R (2011) Practising coarse orientation discrimination improves orientation signals in macaque cortical area V4. Curr Biol 21: 1661-1666. CrossRef Medline

Ahissar M, Hochstein S (1997) Task difficulty and the specificity of perceptual learning. Nature 387:401-406. CrossRef Medline

Ahissar M, Hochstein S (2004) The reverse hierarchy theory of visual perceptual learning. Trends Cogn Sci 8:457-464. CrossRef Medline

Brainard DH (1997) The Psychophysics Toolbox. Spat Vis 10:433-436. CrossRef Medline

Dosher BA, Lu ZL (1998) Perceptual learning reflects external noise filtering and internal noise reduction through channel reweighting. Proc Natl Acad Sci U S A 95:13988-13993. CrossRef Medline

Dosher BA, Jeter P, Liu J, Lu ZL (2013) An integrated reweighting theory of perceptual learning. Proc Natl Acad Sci USA 110:13678-13683. CrossRef Medline 
Fahle M (2004) Perceptual learning: a case for early selection. J Vis 4(10): 879-890. CrossRef Medline

Ghose GM, Yang T, Maunsell JH (2002) Physiological correlates of perceptual learning in monkey V1 and V2. J Neurophysiol 87:1867-1888. Medline

Harris H, Gliksberg M, Sagi D (2012) Generalized perceptual learning in the absence of sensory adaptation. Curr Biol 22:1813-1817. CrossRef Medline

Hua T, Bao P, Huang CB, Wang Z, Xu J, Zhou Y, Lu ZL (2010) Perceptual learning improves contrast sensitivity of V1 neurons in cats. Curr Biol 20:887-894. CrossRef Medline

Hung SC, Seitz AR (2011) Retrograde interference in perceptual learning of a peripheral hyperacuity task. PLoS One 6:e24556. CrossRef Medline

Jehee JF, Ling S, Swisher JD, van Bergen RS, Tong F (2012) Perceptual learning selectively refines orientation representations in early visual cortex. J Neurosci 32:16747-16753a. CrossRef Medline

Jeter PE, Dosher BA, Petrov A, Lu ZL (2009) Task precision at transfer determines specificity of perceptual learning. J Vis 9(3):1.1-13. CrossRef Medline

Jeter PE, Dosher BA, Liu SH, Lu ZL (2010) Specificity of perceptual learning increases with increased training. Vision Res 50:1928-1940. CrossRef Medline

Kahnt T, Grueschow M, Speck O, Haynes JD (2011) Perceptual learning and decision-making in human medial frontal cortex. Neuron 70:549559. CrossRef Medline

Karni A, Sagi D (1991) Where practice makes perfect in texture discrimination: evidence for primary visual cortex plasticity. Proc Natl Acad Sci U S A 88:4966-4970. CrossRef Medline

Law CT, Gold JI (2008) Neural correlates of perceptual learning in a sensory-motor, but not a sensory, cortical area. Nat Neurosci 11:505-513. CrossRef Medline

Le Dantec CC, Seitz AR (2012) High resolution, high capacity, spatial specificity in perceptual learning. Front Psychol 3:222. CrossRef Medline

Li W, Piëch V, Gilbert CD (2004) Perceptual learning and top-down influences in primary visual cortex. Nat Neurosci 7:651-657. CrossRef Medline

Li W, Piëch V, Gilbert CD (2008) Learning to link visual contours. Neuron 57:442-451. CrossRef Medline

Pelli DG (1997) The VideoToolbox software for visual psychophysics: transforming numbers into movies. Spat Vis 10:437-442. CrossRef Medline

Pilly PK, Seitz AR (2009) What a difference a parameter makes: a psychophysical comparison of random dot motion algorithms. Vision Res 49: 1599-1612. CrossRef Medline

Pilly PK, Grossberg S, Seitz AR (2010) Low-level sensory plasticity during task-irrelevant perceptual learning: evidence from conventional and double training procedures. Vision Res 50:424-432. CrossRef Medline

Schoups AA, Vogels R, Orban GA (1995) Human perceptual learning in identifying the oblique orientation: retinotopy, orientation specificity and monocularity. J Physiol 483:797-810. Medline

Schoups A, Vogels R, Qian N, Orban G (2001) Practising orientation identification improves orientation coding in V1 neurons. PG-549-553. Nature 412:549-553. CrossRef Medline

Sotiropoulos G, Seitz AR, Seriés P (2011) Changing expectations about speed alters perceived motion direction. Curr Biol 21:R883-R884. CrossRef Medline

Vaina LM, Belliveau JW, des Roziers EB, Zeffiro TA (1998) Neural systems underlying learning and representation of global motion. Proc Natl Acad Sci U S A 95:12657-12662. CrossRef Medline

Xiao LQ, Zhang JY, Wang R, Klein SA, Levi DM, Yu C (2008) Complete transfer of perceptual learning across retinal locations enabled by double training. Curr Biol 18:1922-1926. CrossRef Medline

Yotsumoto Y, Watanabe T, Sasaki Y (2008) Different dynamics of performance and brain activation in the time course of perceptual learning. Neuron 57:827-833. CrossRef Medline

Zhang GL, Cong LJ, Song Y, Yu C (2013) ERP P1-N1 changes associated with Vernier perceptual learning and its location specificity and transfer. J Vis 13(4):19. CrossRef Medline

Zhang JY, Kuai SG, Xiao LQ, Klein SA, Levi DM, Yu C (2008) Stimulus coding rules for perceptual learning. PLoS Biol 6:e197. CrossRef Medline

Zhang JY, Wang R, Klein SA, Levi DM, Yu C (2011) Perceptual learning transfers to untrained retinal locations after double training: a piggyback effect. J Vis 11(11):1026. CrossRef

Zhang JY, Zhang GL, Xiao LQ, Klein SA, Levi DM, Yu C (2010) Rule-based learning explains visual perceptual learning and its specificity and transfer. J Neurosci 30:12323-12328. CrossRef Medline

Zhang T, Xiao LQ, Klein SA, Levi DM, Yu C (2010) Decoupling location specificity from perceptual learning of orientation discrimination. Vision Res 50:368-374. CrossRef Medline 